\title{
Indicadores de envejecimiento en personas con discapacidad. La percepción de las familias
}

\author{
Catalina Guerrero Romera \\ Universidad de Murcia (España)
}

\begin{abstract}
El artículo presenta los resultados de una investigación sobre la percepción que los familiares de personas mayores con discapacidad intelectual tienen en relación al proceso de envejecimiento $\mathrm{y}$, particularmente, de los indicios más significativos o relevantes que empiezan a detectar en sus familiares en áreas como la cognitiva, emocional, social o laboral y acerca de sus preocupaciones sobre el futuro. Se ha utilizado el método cuantitativo, concretamente tipo encuesta a través del diseño de un instrumento de elaboración propia y sometido a un proceso de validación por expertos. En cuanto a los resultados obtenidos, las áreas que más preocupan son las referidas a aspectos emocionales y sociales, donde los indicios o síntomas percibidos por los familiares en sus hijos e hijas han obtenido puntuaciones mayores, especialmente los relacionados con el aumento de las preocupaciones como pérdida de familiares y amigos, temor a la aparición de enfermedades, a la propia muerte, seguidos de los cambios en la convivencia de cara al futuro. Igualmente, los referidos al bienestar económico o material y a poder tener un centro que potencie su autonomía al máximo, son para las familias aspectos prioritarios.
\end{abstract}

Palabras clave: Indicadores de envejecimiento, discapacidad intelectual, envejecimiento activo, familias, calidad de vida.

Indicators of aging in people with disabilities. The perception of families. The article presents the results of a research on the perception that the relatives of elderly people with intellectual disabilities have in relation to the aging process and, particularly, of the most significant or relevant signs that they begin to detect in their relatives in areas such as cognitive, emotional, social or work and about their worries about the future. The quantitative method has been used, specifically type survey through the design of an instrument of own elaboration and subjected to a process of validation by experts. Regarding the results obtained, the areas of greatest concern are those related to emotional and social aspects, where the signs or symptoms perceived by family members in their sons and daughters have obtained higher scores, especially those related to the increase in concerns such as loss of family and friends, fear of the appearance of illnesses, death itself, followed by changes in coexistence in the future. Likewise, those referred to the economic or material well-being and to be able to have a center that maximizes their autonomy, are priority for families.

Keywords: Active ageing, Indicators of aging, intellectual disability, families, quality of life.

Correspondencia: Catalina Guerrero Romera. Universidad de Murcia. Facultad de Educación. Campus Espinardo. C.P.:30100. Murcia (España). E-mail: cgromera@um.es 
Distintos estudios nos alertan del incremento de la población de personas mayores con discapacidad o en proceso de envejecimiento debido a los avances en bienestar, salud y calidad de vida que han aumentado las expectativas de vida (Berjano y García, 2009; Flórez, 2010; Fernández-Olaria et al., 2011; Del Barrio y Santurce, 2013). Es necesario por tanto anticipar las demandas de este grupo de población y fomentar servicios y recursos adecuados para un envejecimiento activo y saludable que partan de la identificación de aquellas áreas en las que pueden empezar a experimentar mayores cambios (Navas et al., 2014).

De forma general el proceso de envejecimiento en las personas con discapacidad intelectual conlleva cambios importantes que afectan a todos los ámbitos de su vida y a la de sus familias (Berjano y García, 2009; FEAPS-Navarra y Fundación para la Calidad y el Desarrollo Social, 2005; Millán et al., 2006). A partir de estos estudios y a modo de síntesis destacamos algunos de estos cambios:

Tabla 1. Cambios experimentados por las personas mayores con DI en su proceso de envejecimiento

\begin{tabular}{|c|c|}
\hline Cambios & Síntomas \\
\hline Cambios físicos & $\begin{array}{l}\text { Afectación de las funciones sensoriales y perceptivas principalmente pérdidas de audición } \\
\text { y visión, difíciles de detectar muchas veces, por la propia discapacidad; otros tales como } \\
\text { cansancio, movilidad más torpe, menos habilidad, aparición de enfermedades crónicas } \\
\text { ligadas al envejecimiento como las cardiovasculares, digestivas, respiratorias. }\end{array}$ \\
\hline Cambios a nivel cognitivo & $\begin{array}{l}\text { Mayor lentitud en el proceso de reacción, alteraciones de memoria, despistes, olvidos, } \\
\text { desorientación, disminución de la capacidad de resolución de problemas, en la atención } \\
\text { así como en la capacidad de lenguaje y expresión, en la capacidad de adquirir nuevas } \\
\text { destrezas, trastornos psíquicos, demencias, psicosis, ritos neuróticos. }\end{array}$ \\
\hline Cambios socioemocionales & $\begin{array}{l}\text { Pérdida de apoyos sociales o incremento de los apoyos sociosanitarios que pueden } \\
\text { dificultar la autonomía, la autoestima, preocupación por la pérdida de familiares y amigos, } \\
\text { soledad, pérdida de actividad laboral u ocupacional (sobre todo al dejar la actividad } \\
\text { laboral, ya que muchas veces las amistades son los compañeros de trabajo), depresiones, } \\
\text { descontento, tristeza, cambios de humor, menor estabilidad emocional que provoca } \\
\text { inseguridad y angustia, cambios en el comportamiento tales como apatía, menor } \\
\text { motivación, aburrimiento, irritabilidad, aislamiento. }\end{array}$ \\
\hline
\end{tabular}

Nota. Fuente: Guerrero, 2010

Cuando se habla de envejecimiento no debe atenderse sólo a un criterio cronológico o funcional, sino que debe comprenderse dentro del modelo de ciclo vital, ya que existe una gran variabilidad en las manifestaciones que pueden representar declives o empeoramientos en la calidad de vida con la consecuente pérdida de autonomía para ellos y para sus familias (Madrigal, 2006; VVAA, 2006; Berjano y García, 2009; Guerrero, 2010; De Barrios et al., 2016). El proceso de envejecimiento en las personas mayores con DI puede convertirse en un proceso más prematuro y de deterioro rápido y repentino en edades más tempranas y que pueden representar declives o empeoramientos en su calidad de vida con la consecuente pérdida de autonomía (Millán et al., 2006).

No olvidemos que estamos ante una doble vulnerabilidad, la de las personas con discapacidad y la de sus familias que están envejeciendo al mismo tiempo (Novell et al., 2008), lo que incrementa las situaciones de vulnerabilidad en estos casos. Es importante detectar y prevenir estos indicios, así como mantener el mayor nivel de 
autonomía y participación social, poniendo especial énfasis en la prevención dentro de la atención a las personas mayores (MEC, 2014).

Galarza y Díaz (2010) señalaban que las familias son un factor clave en la promoción y el desarrollo de un envejecimiento activo y saludable, ya que son un elemento o unidad de apoyo imprescindible en la atención, cuidado y apoyo a la persona con discapacidad intelectual. Los familiares en la mayoría de los casos asumen los cuidados principales de las personas mayores con discapacidad y constituyen un apoyo primordial para las mismas (Badía, 2005; Berzosa, 2013; Signo et al., 2016). Asimismo, Gómez et al. (2015, p.2) destacaban que "la familia es el contexto más habitual en el que las personas se desarrollan y por tanto se recuperan, con lo que es necesario entenderla con potencialidades y recursos propios y útiles para atender los procesos vitales de sus miembros". Son por tanto numerosos los autores que subrayan la importancia que tiene extraer la información del contexto familiar para documentar los cambios que se pueden estar produciendo (Signo et al., 2016).

Las diversas aportaciones desde el modelo social de la discapacidad también señalan el papel que tienen los distintos factores socioculturales entre los que se encuentran las características de las familias y las necesidades de contar con los apoyos adecuados que eviten problemas de salud (Venturiello, 2014). López et al. (2017) señalaban asimismo las dificultades y cargas que las familias deben afrontar en la esfera privada en relación a los cuidados que deben asumir, lo que requiere indagar en las percepciones de los entornos familiares y en los roles de cuidado tradicionales de la familia para una adecuada intervención que aseguren el mantenimiento de la mayor calidad de vida posible.

Por otra parte, desde el enfoque de calidad de vida de Park, Turnbull, y Turnbull (2002) el papel de la familia es uno de los factores más destacados. Se entiende que la Calidad de Vida Familiar queda comprendida por cinco factores como la dimensión social y emocional y los propios recursos familiares para afrontar y apoyar a la persona con discapacidad intelectual, unido al propio papel que tiene la familia.

El modelo de envejecimiento activo de la OMS (2002) está basado precisamente en una serie de pilares (salud, participación social y seguridad) y determinantes de los mismos (económicos, sociales, personales,...) que destacan las relaciones entre actividad, independencia, autonomía y la posibilidad de mantener una buena calidad de vida. En esta misma línea, la OCDE también destacó 11 dimensiones básicas para medir la calidad de vida entre las que se encuentran las variables ingresos, salud y satisfacción ante la vida. Igualmente, Verdugo et al. (2013, p. 26) destacaron la variable familias como una de las variables que tenía o presenta mayor relación positiva con su calidad de vida.

La finalidad del estudio ha sido por tanto analizar la percepción de los familiares de personas mayores con discapacidad intelectual o en proceso de 
envejecimiento sobre los indicios o síntomas que están detectando en dicho proceso y acerca de sus preocupaciones. En definitiva, evaluar la percepción que tienen sobre el proceso de envejecimiento y sobre los indicios que están percibiendo en relación a las áreas cognitiva, emocional y social, física, salud mental y laboral y que pueden ser indicadores de un inicio temprano de los procesos de envejecimiento.

\section{MÉTODO}

Se trata de una investigación de carácter cuantitativo no experimental y de corte descriptivo tipo encuesta.

\section{Participantes}

Los participantes en este estudio han sido 47 familias que cumplimentaron una encuesta que permitió el análisis de los indicadores más relevantes que estaban percibiendo en sus hijos/as o familiares con discapacidad desde su propia perspectiva, así como otras preocupaciones y cuestiones sobre el proceso de envejecimiento. Los familiares participantes pertenecían a una entidad dirigida a la atención y promoción de la autonomía personal del colectivo de personas con discapacidad intelectual. Esta entidad fue seleccionada ya que es pionera en la creación y desarrollo del primer Servicio de Promoción de la Autonomía Personal, y en la que sus diferentes áreas (Empleo, Educación, Tránsito al Mundo Laboral, Formación Integral y Continuada....) están encaminadas a conseguir la máxima autonomía e independencia.

Dicha entidad, además cuenta con un número considerable de personas mayores o en proceso de envejecimiento lo que es un elemento clave para la realización de la investigación y para la realización de propuestas de intervención futuras en esta organización.

Las características más destacables de los participantes son las siguientes. En cuanto al Género, el 64\% $(n=30)$ de los familiares participantes en esta investigación son mujeres frente al 34\% ( $n=16)$ compuesto por hombres (Tabla 1).

Tabla 2. Participantes según género

\begin{tabular}{|c|c|c|c|}
\hline & & Frecuencia & Porcentaje \\
\hline \multirow{3}{*}{ Válidos } & Femenino & 30 & 64 \\
\hline & Masculino & 16 & 34 \\
\hline & Total & 46 & 98 \\
\hline Perdidos & Sistema & 1 & 2 \\
\hline Total & & 47 & 100 \\
\hline
\end{tabular}


En relación a la Edad, un $54 \%$ ( $n=24)$ lo constituyen personas con más de 60 años, un 38\% $(n=17)$ entre 46-59 años y sólo el $9 \%(n=4)$ de los familiares tienen entre 31-45 años (Tabla 2), con una media de edad de 58.94 años.

Tabla 3. Participantes según edad

\begin{tabular}{|c|c|c|c|}
\hline & & Frecuencia & Porcentaje \\
\hline \multirow[t]{4}{*}{ Válidos } & $31-45$ años & 4 & 9 \\
\hline & 46-59 años & 17 & 38 \\
\hline & Más de 60 & 24 & 54 \\
\hline & Total & 45 & 100 \\
\hline Perdidos & Sistema & 2 & 4 \\
\hline Total & & 47 & 100 \\
\hline
\end{tabular}

En cuanto, a los Niveles de Estudios, el 34\% ( $n=16)$, son familiares con estudios de Bachiller o FP, el 26\% ( $n=12)$, posee estudios de primaria, el 19\% ( $n=9)$, tiene estudios universitarios, el 15\% ( $n=7)$, tiene el nivel de ESO y por último un $4 \%(n=2)$ de los familiares no posee ningún tipo de estudios. Con respecto a la Ocupación Laboral, el $43 \%$ $(n=20)$ está en activo, el 34\% ( $n=16)$, son jubilados y el 19\% (n=9), de los familiares se encuentran en desempleo.

Respecto, al Vínculo familiar, el 79\% $(n=37)$ de los participantes son Madre o Padre de la persona con discapacidad; el $15 \%(n=7)$ tiene un vínculo familiar de hermano/a, el 4\% $(n=2)$ se corresponde a la categoría de otros (Tutor/a...) y sólo un 2\% $(n=1)$ abuelo/a. En cuanto, al Estado Civil, el 58\% ( $n=28)$ son Casados/as; el 16\% $(n=7)$, son Divorciados/as; el 15\% $(n=7)$, son Solteros/as, y por último el $11 \%(n=5)$, son viudos/as.

\section{Instrumento y procedimiento}

Se ha utilizado un cuestionario de elaboración propia en base a las dimensiones del modelo de calidad de vida de Schalock y Verdugo (2003), y sometido a un proceso de validación por expertos. Los mismos valoraron el cuestionario de manera muy positiva, obteniendo valoraciones altas, ninguna con una media inferior a 3 en la escala Likert. De esta forma, atendiendo a las puntualizaciones y propuestas de mejora planteadas por los expertos, se llevó a cabo la elaboración definitiva del cuestionario.

El cuestionario utilizado ha consistido en una escala autoaplicada que consta de 72 ítems tipo Likert con una escala de respuesta de 4 grados de intensidad (1: Nada; 2: Poco; 3: Bastante; 4: Mucho). Los ítems han recogido información sobre:

-Datos de identificación (edad, género, ocupación laboral, $n^{\circ}$ de hijos o personas a su cargo, localidad, vínculo familiar y estado civil).

-Indicios de envejecimiento en una serie de áreas como la cognitiva, emocional y social, física, salud mental y laboral.

-Preocupaciones sobre el futuro. 
-Temas y actividades considerados importantes en materia de envejecimiento para los familiares.

Su fiabilidad obtuvo un Alpha de Crombach de .817. Los datos obtenidos han sido tratados a través de técnicas estadísticas, en este caso, el programa IBM SPSS Statistics for Windows, Versión 19.0.

\section{RESULTADOS}

A continuación, se exponen en primer lugar los resultados obtenidos del análisis del cuestionario de percepción de las familias de personas con discapacidad sobre el proceso de envejecimiento y sobre los indicios de envejecimiento más significativos o relevantes que los familiares están empezando a detectar en sus familiares con discapacidad en relación a las áreas cognitiva, emocional, física, salud mental y laboral.

Respecto a los indicios de envejecimiento que los familiares están percibiendo en relación al Área Cognitiva (Tabla 3), en general no se muestran porcentajes muy elevados siendo los indicios detectados con puntuaciones más altas: la dificultad para la toma de decisiones con un 29\% ( $n=11)$ (media 2.61; Desv 1.152; CV 0.441); mayor lentitud para dar respuesta con un 26\% ( $n=10$ ) (media 2.42; Desv 1.154; CV 0.477); las alteraciones de memoria y olvidos, con un 22\% $(n=8)$ (media 2.27; Desv 1.194; CV 0.525) y las dificultades de concentración y atención con un 18\% $(n=7)$ (media 2.37; Desv 1.076; CV 0.454).

Tabla 4. Área Cognitiva

\begin{tabular}{|c|c|c|c|c|c|c|c|c|}
\hline \multirow{2}{*}{ ÁREA COGNITIVA } & \multicolumn{2}{|c|}{ Nada } & \multicolumn{2}{|c|}{ Poco } & \multicolumn{2}{|c|}{ Bastante } & \multicolumn{2}{|c|}{ Mucho } \\
\hline & $n$ & $\%$ & $n$ & $\%$ & $n$ & $\%$ & $n$ & $\%$ \\
\hline Alteraciones de la memoria y olvidos & 14 & $38 \%$ & 7 & $19 \%$ & 8 & $22 \%$ & 8 & $22 \%$ \\
\hline $\begin{array}{l}\text { Disminución de la capacidad de reacción y de resolución de problemas ante } \\
\text { situaciones y dificultades de la vida cotidiana }\end{array}$ & 8 & $21 \%$ & 12 & $31 \%$ & 13 & $33 \%$ & 6 & $15 \%$ \\
\hline $\begin{array}{l}\text { Pérdida de las capacidades sensoriales y perceptivas (vista, oído, gusto, tato } \\
\text { y olfato) }\end{array}$ & 16 & $42 \%$ & 13 & $34 \%$ & 3 & $8 \%$ & 6 & $16 \%$ \\
\hline Dificultad para el reconocimiento de personas y espacios & 25 & $74 \%$ & 5 & $15 \%$ & 1 & $3 \%$ & 3 & $9 \%$ \\
\hline Dificultades con la memoria y el lenguaje & 15 & $43 \%$ & 11 & $31 \%$ & 4 & $11 \%$ & 5 & $14 \%$ \\
\hline $\begin{array}{l}\text { Disminución de la capacidad de concentración y atencional (problemas de } \\
\text { atención, concentración) }\end{array}$ & 10 & $26 \%$ & 11 & $29 \%$ & 10 & $26 \%$ & 7 & $18 \%$ \\
\hline Dificultad para la toma de decisiones & 9 & $24 \%$ & 8 & $21 \%$ & 10 & $26 \%$ & 11 & $29 \%$ \\
\hline $\begin{array}{l}\text { Pérdida o disminución de las habilidades académicas: capacidad de cálculo, } \\
\text { dificultades de lectoescritura,... }\end{array}$ & 10 & $29 \%$ & 9 & $26 \%$ & 11 & $31 \%$ & 5 & $14 \%$ \\
\hline Mayor lentitud para dar respuesta & 10 & $26 \%$ & 12 & $32 \%$ & 6 & $16 \%$ & 10 & $26 \%$ \\
\hline Pérdida de capacidad para realizar tareas complejas & 12 & $32 \%$ & 10 & $27 \%$ & 11 & $30 \%$ & 4 & $11 \%$ \\
\hline $\begin{array}{l}\text { Dificultades para la orientación en el tiempo y el espacio o para la } \\
\text { realización de actividades de la vida diaria }\end{array}$ & 18 & $47 \%$ & 6 & $16 \%$ & 8 & $21 \%$ & 6 & $16 \%$ \\
\hline
\end{tabular}

En cuanto al Área Emocional y Social, los indicios más significativos percibidos por las familias están relacionados con el aumento de las preocupaciones como pérdida de familiares y amigos, temor a la aparición de enfermedades, a la propia muerte, a la pérdida de la actividad laboral y ocupacional, con un 54\% ( $n=20)$ (media 2.54; Desv 
1.169; CV 0.460); los cambios en la convivencia de cara al futuro: residencia, piso tutelado, etc. con un 49\% ( $n=17$ ) (media 2.46; Desv 1.221; CV 0.496); menor participación en actividades sociales, de ocio y tiempo libre, 45\% ( $n=16)$ (media 2.34; Desv 1.192; CV 0.509); y la disminución en la autoestima al realizar menos actividades 44\% ( $n=15)$ (media 2.19; Desv 1.101; CV 0.503). Resulta significativo que un $46 \%(n=17)$ (media 2.05; Desv 1.153; CV 0.562) indicaron que no habían detectado sentimientos de soledad, desamparo y tendencia al aislamiento en sus familiares con discapacidad aunque sí habían detectado mayores preocupaciones sobre los aspectos anteriormente mencionados.

De los indicios del Área Física o de bienestar físico, las familias señalaron como el más significativo que estaban detectando, el aumento de peso, con un $48 \% \quad(n=16)$ (media 2.41; Desv 1.158; CV 0.481), los demás han obtenido unas puntuaciones bastante bajas y van relacionados principalmente con dificultades en la alimentación, el sueño y la osteoporosis.

En el Área de Salud mental, los indicios mayormente detectados por los familiares están relacionados con la alteración de los comportamientos o conductas, un 30\%, (n=14) (media 2.19; Desv 0.995; CV 0.454); problemas en la regulación emocional (aparición o cambios): impulsividad, ira, violencia, cambios de humor bruscos, con un 26\% ( $n=12$ ) (media 2.09; Desv 1.04: CV 0.498); los soliloquios con un 26\% ( $n=12)$ (media 1.9; Desv 1.165; CV 0.613); o la aparición de rasgos de tipo neurótico: angustias, fobias, obsesiones, con un 21\% ( $n=11$ ) (media 2.14; Desv 0.974; CV 0.455). Los indicios relacionados con depresión, síntomas de demencia o alucinaciones fueron los que señalaron con una menor frecuencia.

Respecto a los indicadores del Área de Actividad Laboral, las puntuaciones mayores se dan en nada o poca prioridad concedida a los ítems de esta área. En cualquier caso, los porcentajes más altos señalados por los familiares en este bloque van referidos a la disminución de la motivación $(40 \%, n=14)$ (media 2.11; Desv 1.183; CV 0.561), las dificultades para el aprendizaje y olvidos $(33 \%, n=12)$ (media 2.06; Desv 1.04; CV 0.505), o la disminución de reflejos o mayor torpeza o fatiga con un $30 \%$ respectivamente $(n=10)$ (media 1.85; Desv 1.004; CV 0.542 y media 2; Desv 1; CV 0.50).

Por otra parte, respecto a los resultados acerca de las Preocupaciones sobre el Futuro por parte de los familiares (Tabla 4), el 74\% (57\% y 17\%) (media 3.11; Desv 1.185; CV 0.381) señalaron como mucha y bastante preocupación respectivamente el indicador "contar con un Servicio específico creado por la fundación: viviendas compartidas de Mayores", seguido de "Vivienda o domicilio familiar con los apoyos externos necesarios" con un $54 \%$ y $30 \%$ (media 3.27, Desv 0.99, CV 0.303). Otra de las más prioritarias destacadas por las familias es "Buscar un centro abierto donde se siga potenciando su autonomía”, un 62 \% (media 3.14, Desv 1.167, CV 0.372) de los familiares destaca que esta cuestión les preocupa especialmente. Por otra parte, señalado con la prioridad más 
alta de la escala un 54\% ( $n=21)$ (media 2.9, Desv 1.314, CV 0.453) destaca "Quién va a ser su tutor legal cuando no estemos nosotros". Por último, el más bajo señalado en este bloque es el indicador "Buscar un servicio residencial en nuestra comunidad" el $30 \%$ $(n=10)$ (media 2.39, Desv 1.273, CV 0.533).

Tabla 5. Preocupaciones sobre el futuro

\begin{tabular}{lcccccccc}
\hline \multirow{2}{*}{ PREOCUPACIONES SOBRE EL FUTURO } & \multicolumn{2}{c}{ Nada } & \multicolumn{2}{c}{ Poco } & \multicolumn{2}{c}{ Bastante } & \multicolumn{2}{c}{ Mucho } \\
\cline { 2 - 9 } & $n$ & $\%$ & $n$ & $\%$ & $n$ & $\%$ & $n$ & $\%$ \\
\hline $\begin{array}{l}\text { Quién va a ser su tutor legal cuando no } \\
\text { estemos nosotros }\end{array}$ & 10 & $26 \%$ & 5 & $12 \%$ & 3 & $8 \%$ & 20 & $54 \%$ \\
\hline $\begin{array}{l}\text { Buscar un centro abierto donde se siga } \\
\text { potenciando su autonomía }\end{array}$ & 6 & $17 \%$ & 3 & $9 \%$ & 6 & $17 \%$ & 21 & $57 \%$ \\
\hline $\begin{array}{l}\text { Buscar un servicio residencial en la } \\
\text { comunidad }\end{array}$ & 12 & $36 \%$ & 6 & $18 \%$ & 5 & $15 \%$ & 10 & $30 \%$ \\
\hline $\begin{array}{l}\text { Que siga viviendo en el domicilio familiar } \\
\text { cuidado por sus hermanos u otros familiares }\end{array}$ & 7 & $22 \%$ & 1 & $3 \%$ & 8 & $25 \%$ & 16 & $50 \%$ \\
\hline $\begin{array}{l}\text { Vivienda o domicilio familiar con los } \\
\text { apoyos externos necesarios }\end{array}$ & 4 & $11 \%$ & 2 & $5 \%$ & 11 & $30 \%$ & 20 & $54 \%$ \\
\hline $\begin{array}{l}\text { Servicio específico creado por la fundación: } \\
\text { viviendas compartidas de Mayores }\end{array}$ & 8 & $18 \%$ & 4 & $1 \%$ & 7 & $16 \%$ & 25 & $57 \%$ \\
\hline \begin{tabular}{l} 
Nota Fuente: Elaboración propia \\
\hline
\end{tabular}
\end{tabular}

Nota. Fuente: Elaboración propia

En este apartado o bloque también se incluyeron algunas cuestiones específicas para conocer las preocupaciones referidas al área Económica (Tabla 5), siendo las más prioritarias para los familiares: "La transición a la pensión de jubilación" con un 72\% $(n=26)$ (media 3.39, Desv 1.076, CV 0.317), "La prestación de ayudas de la Ley de Dependencia" con un 66\% ( $n=27$ ) (media 3.46, Desv 0.897, CV 0.259), le sigue "La cuantía de las pensiones o retribuciones que recibirá mi familiar" con un $65 \%(n=26)$ (media 3.43, Desv 0.903, CV 0.263); y, por último, el ítem "La situación económica familiar" con un 53\% ( $n=18)$ (media 3.26, Desv 0.931, CV 0.286), siendo esta área de bienestar económico y material una de las que ha obtenido las medias más altas.

Tabla 6. Área Económica

\begin{tabular}{|c|c|c|c|c|c|c|c|c|}
\hline \multirow{2}{*}{ ÁREA ECONÓMICA } & \multicolumn{2}{|c|}{ Nada } & \multicolumn{2}{|c|}{ Poco } & \multicolumn{2}{|c|}{ Bastante } & \multicolumn{2}{|c|}{ Mucho } \\
\hline & $n$ & $\%$ & $n$ & $\%$ & $n$ & $\%$ & $n$ & $\%$ \\
\hline La cuantía de las pensiones o retribuciones que recibirá mi familiar & 2 & $5 \%$ & 5 & $12 \%$ & 7 & $18 \%$ & 26 & $65 \%$ \\
\hline La prestación o ayudas de la Ley de Dependencia & 3 & $7 \%$ & 2 & $5 \%$ & 9 & $22 \%$ & 27 & $66 \%$ \\
\hline La transición a la pensión de jubilación & 4 & $11 \%$ & 4 & $11 \%$ & 2 & $6 \%$ & 26 & $72 \%$ \\
\hline La situación económica familiar & 2 & $6 \%$ & 5 & $15 \%$ & 9 & $26 \%$ & 18 & $53 \%$ \\
\hline
\end{tabular}

Finalmente, y en relación a qué temas y actividades los familiares consideran importantes en materia de envejecimiento, la mayoría de los planteados en el cuestionario han sido percibidos por los familiares como muy prioritarios, aunque destacamos a continuación los de mayor porcentaje y que han obtenido las puntuaciones más altas de la escala: "Los Testamentos vitales" con un 90\% (n=38) (media 3.78, Desv 0.722, CV $0.191)$, "Respeto a su forma de vida y a su autonomía", con un 88\% ( $n=36)$ (media 3.84, 
Desv 0.531, CV 0.138), “Toma de decisiones de nuestros hijos cuando sean mayores” con un 87\% ( $n=34$ ) (media 3.87, Desv 0.339, CV 0.088), "Detección de los signos de deterioro y su diagnóstico" con un 77\% $(n=30)$ (media 3.53, Desv 0.96, CV 0.272) y Habilidades emocionales e inteligencia emocional con un 66\% $(n=27)$ (media 3.56, Desv 0.673, CV 0.189 ). Los familiares se muestran especialmente preocupados por aspectos relacionados con la autonomía y la toma de decisiones.

\section{DISCUSIÓN Y CONCLUSIONES}

Los resultados obtenidos en esta investigación nos muestran algunos de los indicios de envejecimiento que las familias están empezando a detectar en sus hijos/as o familiares con discapacidad, así como algunas de sus preocupaciones e incertidumbres sobre el futuro de los mismos. En relación a los indicios de envejecimiento detectados por los familiares, es en el área emocional y social donde se han obtenido puntuaciones más altas. Respecto a los indicios o síntomas percibidos por los familiares en esta área se han obtenido las puntuaciones mayores, especialmente en los relacionados con el aumento de las preocupaciones como pérdida de familiares y amigos, temor a la aparición de enfermedades, a la propia muerte, seguidos de los cambios en la convivencia de cara al futuro: residencia, piso tutelado, coincidiendo con investigaciones recientes como las de Signo et al. (2016).

En la investigación de Lantegi Batuak (2011, p. 11) se señalaron asimismo como destacables "los sentimientos de incertidumbre ante el futuro o de tristeza y duelo ante las situaciones de envejecimiento/deterioro de las familias". Los familiares de nuestro estudio indicaron que no habían detectado sentimientos de soledad, desamparo y tendencia al aislamiento en sus familiares con discapacidad, algo señalado en la investigación de Aguado (s.f.) respecto a las necesidades de las personas con discapacidad intelectual en proceso de envejecimiento, ya que no tienen una visión negativa del futuro, ni tendencia al aislamiento ni a la soledad (p. 29).

Del mismo modo, las áreas relacionadas con la salud y la autonomía son otras de las constantes preocupaciones destacadas por los familiares confirmados también en el estudio de Aguado (s.f.). El bienestar emocional, la salud física y la salud mental han sido asimismo destacados por las familias que han encontrado indicios en problemas de comportamiento, cambio de conductas o problemas de regulación emocional, coincidiendo también con otros estudios que arrojan resultados en este sentido (Farriols, 2012; Flórez, 2010; Millán Callenti et al., 2006; Novell, 2008; Signo et al., 2016).

En nuestra opinión la implicación familiar debe contemplarse en esta identificación de los indicios de envejecimiento máxime si tenemos en cuenta el papel o rol principal que la familia tiene en la atención, cuidado y apoyo a la persona con discapacidad intelectual (Badía, 2002; CERMI, 2012; Fernández et. al., 2011; Park, 
Turnbull, y Turnbull, 2002; Schalock y Verdugo, 2003). De hecho, el modelo de la calidad de vida familiar de Fernández et al. 20011 destaca varios factores entre los que se encuentran algunos de los contemplados en nuestra investigación y que han sido percibidos igualmente por los participantes como importantes: bienestar emocional, interacción familiar, participación social, bienestar económico y salud.

En cualquier caso, estos factores deben contemplarse en función de las edades y de los contextos, y de la percepción sobre el "grado de satisfacción experimentado por la persona en relación con el nivel de cobertura de sus necesidades en el entorno que le rodea: en el hogar, en la escuela, en el trabajo y, en definitiva, en la comunidad" (Giné, 2004 , p. 20). Por esta razón se han de evaluar de forma particular dichas necesidades, en relación a estos parámetros, a partir de las percepciones y valores más subjetivos y de las condiciones de vida más objetivas como apuntó (Aby Analistas y Atades, 2013; Giné, 2004, p. 21), lo que hacen necesarias más investigaciones que tenga en cuenta la perspectiva de las familias.

Los resultados corroboran otras investigaciones existentes que también ponen de manifiesto cómo la incertidumbre con respecto al futuro del familiar con discapacidad da lugar a la aparición de nuevas demandas por parte de los cuidadores principales: pensiones, retribuciones o prestaciones que pueden percibir las personas con discapacidad o la búsqueda de servicios y apoyos específicos u otras fórmulas que se están desarrollando en los últimos años (Aguado, s.f; González del Yerro et al., 2013; Rodríguez et al., 2008). En nuestra investigación son precisamente las variables relacionadas con el bienestar económico y material las que han obtenido puntuaciones más altas.

La mayoría de los padres son los cuidadores principales de la persona con discapacidad intelectual que envejece, estos están a la vez haciendo frente a su propio proceso de envejecimiento. Si tenemos en cuenta los datos de nuestro estudio, más de la mitad de los familiares son personas con más de 60 años y sólo un $9 \%$ eran menores de 45 años, siendo un 34\% de ellos jubilados, por lo que se deberán proporcionar los apoyos necesarios para que sean capaces de adaptarse tanto a los cambios asociados al envejecimiento de su hijo/a como al suyo propio (Navas et al., 2014).

En nuestro estudio las preocupaciones mayores sobre el futuro por parte de los familiares son "Buscar un centro abierto donde se siga potenciando su autonomía", siendo esta cuestión un aspecto fundamental, como se puede apreciar en la importancia que le conceden a que se respete su forma de vida y su autonomía al máximo posible, por lo que habrá que desarrollar fórmulas que permitan avanzar en este sentido: hogares o residencias compartidas, apoyos en el propio hogar, nuevas iniciativas de respiro familiar,..., ya que aún hay una falta o escasez de especialización y servicios en temas de envejecimiento y discapacidad (Berzosa et al., 2013; Rodríguez et al., 2008). Los datos obtenidos muestran algunas de las inquietudes de las familias, especialmente preocupadas por el futuro de los 
hijos/as cuando ellos no estén y el que existan servicios que puedan atenderles y continuar un proceso de envejecimiento activo y saludable.

Igualmente, una de las preocupaciones fundamentales de las familias es que se realice una detección y diagnóstico de los signos de deterioro a través de escalas o dispositivos de valoración técnica_que permitan una adecuada intervención y prevención. En este sentido, resulta prioritario poder incluir estas valoraciones como elementos de análisis y diagnóstico, así como establecer programas de intervención que contribuyan a prevenir el avance de algunos de estos síntomas y permitan mantener al máximo los niveles de autonomía y participación social (Signo et al., 2016; MEC, 2014; NDSS, 2013; Guerrero, 2010; Millán et al., 2006). Dichas valoraciones deberían realizarse de modo más continuo para poder responder a las especificidades y cambios que puedan experimentar (Observatorio de la Discapacidad, 2015).

En consonancia con lo anterior y teniendo en cuenta el análisis de los resultados de las inquietudes y necesidades sobre el proceso de envejecimiento de sus hijos/as o familiares con discapacidad intelectual, las familias están interesadas asimismo en temas y actividades para poder afrontar con éxito y con la mayor normalidad posible esta nueva etapa vital, ya que el desconocimiento de muchos indicadores o características del proceso de envejecimiento de su hijo/a mayor con discapacidad, les provoca mucha incertidumbre e inseguridad (Berjano y García, 2009; NDSS, 2013; Del Barrio et al., 2016).

En la investigación se han detectado como temas de interés y preocupación para ellos, la necesidad de detección de los signos de deterioro y diagnóstico temprano; la toma de decisiones de los hijos mayores con discapacidad intelectual; respeto a su forma de vida y a su autonomía, quién lo respetará si no está en sus plenas facultades y testamentos vitales. En definitiva, todo aquello que conlleve el máximo desarrollo de la persona y la potenciación de sus capacidades de elección, autodeterminación en aras de un envejecimiento no sólo activo y saludable, sino también lo más autónomo posible.

En consecuencia es necesaria la identificación de las áreas que necesitan más apoyo y que en nuestro estudio apuntan fundamentalmente a las áreas emocionales y de participación social, pero también a las relacionadas con el bienestar material y económico por encima de otros aspectos como los cognitivos, en la línea de algunas de las dimensiones destacadas por el modelo de envejecimiento activo de la OMS (2002). López et al. (2017) también destacan el papel que tienen las aportaciones económicas al hogar junto con otras como el desempeño de las tareas y actividades básicas.

Por otra parte, Núñez, Rodríguez, y Lanciano (2005) afirman que los padres enfrentan una verdad para ellos muy angustiante: el propio envejecimiento y el miedo a la propia muerte; la dolorosa realidad de no poder "ya estar presentes" cuando su hijo lo requiera, y la "permanente duda de si los otros estarán cuando su hijo lo necesite". Respecto a ello, los resultados de nuestra investigación corroboran que los familiares muestran mucha inseguridad sobre el futuro de su familiar mayor con discapacidad 
(Aguado, s.f.) que en este caso además si las comparamos con otra investigación acerca de las necesidades sobre el proceso de envejecimiento percibido por profesionales de la atención especializada a personas con discapacidad, son coincidentes con las familias en cuanto a la prioridad concedida a áreas como autonomía, vida independiente y desarrollo personal, junto con el bienestar socioemocional y el bienestar físico (Guerrero, 2018, p. 79), coincidiendo asimismo estos resultados con otra investigación sobre la percepción de los familiares y tutores de personas con discapacidad en la que también señalan como dimensiones más significativas en la calidad de vida, el bienestar emocional y las relaciones interpersonales (Aby Analistas y Atades, 2013, p. 34).

Los resultados de dicha investigación subrayaban la importancia que los profesionales de atención directa otorgan a las cuestiones relacionadas con la promoción de la autonomía y vida independiente y el desarrollo emocional y sociopersonal, aspectos relacionados con la promoción de la salud, la calidad de vida, la prevención y el envejecimiento activo de las personas mayores con discapacidad intelectual en situación de especial vulnerabilidad en la línea de algunos informes especializados (Ministerio de Educación, Cultura y Deporte, 2014). En esta promoción de un envejecimiento activo y saludable, las familias tienen una función esencial, ya que son una de las principales redes de apoyo de las personas mayores con DI.

Es necesario, por tanto, identificar aquellos indicios o síntomas que se están detectando y empezar a trabajar con ellos, así como realizar más estudios de necesidades en el contexto familiar que permitan establecer los apoyos y servicios necesarios identificados también por las familias en relación al proceso de envejecimiento. Consideración que ya recogía el Observatorio Estatal de la Discapacidad en su informe sobre la situación del envejecimiento de las personas con discapacidad en el año 2015. No obstante, como apuntaron Giné et al. (2015, p. 87) "el peso no va a estar tanto en el diagnóstico, sino en sus consecuencias para la intervención”.

\section{Agradecimientos}

Queremos expresar nuestro agradecimiento a las familias participantes en la realización de este estudio y a la entidad a la que pertenecen por su implicación en el mismo.

\section{REFERENCIAS}

Aguado, A. (Dir.) (s.f). Necesidades de las personas con discapacidad intelectual en proceso de envejecimiento. Recuperado de http://www.ifas.bizkaia.eus/fitxategiak/dokumentuak/ Necesidades\%20ppdd.pdf?hash=c0660fa4fdd646377bd77f80fe6d01fc

Abay Analistas y Atades (2013). Primer panel de indicadores de calidad de vida de personas con discapacidad en Aragón. Recuperado de http://www.atades.com/wp- 
content/uploads/2013/12/Primer-panel-de-indicadores-de-calidad-de-vida-de-personascon-discapacidad-intelectual-en-Aragon-ATADES.pdf

Badía I., y Corbella, M. (2005). Calidad de vida familiar: La familia como apoyo a la persona con parálisis cerebral. Intervención Psicosocial, 14(3), 327-341.

Berjano, E., y García, E. (2010). Discapacidad intelectual y envejecimiento. Un problema social del siglo XXI. Madrid: Colección FEAPS

Berzosa, G. (dir.) (2013). Las personas con síndrome de Down y sus familias ante el proceso de envejecimiento. Madrid: Real Patronato sobre Discapacidad.

Del Barrio, J.A., Sánchez, A., y González-Santos, J. (2016). Retos del envejecimiento de las personas con discapacidad intelectual. International Journal of Developmental and Eduactional Psychology, 2, 47-56.

Del Barrio J.A., y Santurce, E. (2013). Retos: discapacidad intelectual y el proceso de envejecimiento. International Journal of Developmental and Eduactional Psychology, 2(1), 17-22.

Farriols, C. (2012). Aspectos específicos del envejecimiento en el síndrome de Down. Revista Médica Internacional sobre el Síndorme de Down 16(1), 3-10.

Fernández-Olaria, R., Signo, S., Bruna, O., y Canals, G. (2011). Envejecimiento activo y saludable en personas con Síndrome de Down. Estudio multicéntrico para la prevención e intervención del deterioro cognitivo. Síndrome Down: Vida Adulta, 9, 87-90.

Flórez, J. (2010). Enfermedad de Alzheimer y síndrome de Down. Revista Síndrome Down, 27(2), 63-76.

Fernández, C., Torrecillas, A.M., Díaz, G., y Florido, J. (2011). Factores de calidad de vida de las familias con personas con discapacidad intelectual: experiencia en Gran Canaria. International Journal of Developmental and Educational Psychology, 1(4), 585-592.

Fernández-Ballesteros, R. (2005) Evaluation of "Vital Aging-M": A Psychosocial Program for promoting Optimal Aging. European Psychologist, 10(2), 146-156.

Galarza, A.A. y Díaz, J.P. (2010). Familia, envejecimiento y discapacidad en España. Papers. Revista de Sociología, 95(3), 673-700.

Giné, C. (2004). Servicios y calidad de vida para las personas con discapacidad intelectual. Revista Española sobre Discapacidad Intelectual, 35(2), 18-28.

Gine, C., Montero, D., Verdugo M.A., Rueda, P., y Vert, S. (2015). Claves de futuro en la atención y apoyo a las personas con discapacidad intelectual y del desarrollo ¿Qué nos dice la ciencia? Siglo Cero, 46(1), 81-106.

Gómez, R., Contreras, M., Toledano, S., y Parrabera, S. (2015). Conclusiones XIII Jornadas de Fundación Manantial bajo el título "Un lugar para la familia". Madrid: Fundación Manantial.

González-del-Yerro, A., Simón-Rueda, C., Cagigal-Gregorio, V., y Blas-Gómez, E. (2013). La calidad de vida de las familias de personas con discapacidad intelectual. Un estudio cualitativo realizado en la comunidad de Madrid. Revista Española de Orientación y Psicopedagogía, 24(1), 93-109.

Guerrero, C. (2010). Procesos de envejecimiento en personas con discapacidad intelectual: una propuesta integradora. Enlace en Red, $\mathrm{n}^{\circ}$ 15, 22-30. Revista de RIICOTEC (Red Intergubernamental Iberoamericana de Cooperación Técnica) Ministerio de Trabajo y Asuntos Sociales.

Guerrero, C. (2018). Necesidades formativas de los profesionales en centros de atención especializada a personas mayores con discapacidad. En Gázquez, J.J; Pérez-Fuentes, $\mathbf{M}^{\mathrm{a}}$ C.; Molero, M M M.; Simón, Ma M.; Martos, A. y Barragán, A. (Comps). Conocimientos, investigación y prácticas en el campo de la salud. Volumen III. Madrid: ASUNIVEP, 7985. 
Lantegi, B. (2011). Investigación sobre el envejecimiento y deterioro de las personas con discapacidad intelectual en el ámbito ocupacional y del empleo en Bizkaia. Bizkaia: Lantegi Batuak.

López-Díaz, A., Florisa, V., Alzate, M., y Bom-Mehyl, J.C. (2017). Dinámicas y prácticas de cuidado en la pobreza, la vejez y la discapacidad: La familia Vargas. Investigación en Enfermería: Imagen y Desarrollo, 19(1), 139-153.

Madrigal, A. (2006). Marco Teórico del Envejecimiento de las Personas con Discapacidad Intelectual. Perfiles y tendencias, Boletín sobre el Envejecimiento, 26, 4-18.

Millán-Calenti, J.C. (2006). Personas mayores con discapacidad intelectual: Propuesta para la puesta en marcha de un programa de intervención y resultados preliminares. Revista Siglo Cero, $37(2), 218$.

Ministerio de Educación, Cultura y Deporte (2014). Envejecimiento activo y discapacidad intelectual. Madrid: Ministerio de Educación, Cultura y Deporte.

Navas, P., Uhlmann, S., y Berasategui, A. (2014). Envejecimiento Activo y Discapacidad Intelectual. Madrid: Ministerio de Educación, Cultura y Deporte. Colección Investigación.

NDSS (2013). Envejecimiento y sindrome de Down. Una guía de salud y bienestar. Recuperado de http://www.ndss.org/

Novell, R., Nadal, M., Smilges, A.P., y Pujol, J. (2008). Informe Séneca. Envejecimiento y discapacidad Intelectual en Cataluña. Barcelona: FEAPS.

Núñez, B., Rodríguez, L., y Lanciano, S. (2005). El vínculo fraterno cuando uno de los hermanos tiene discapacidad. Revista Española sobre Discapacidad Intelectual, 36(4), 50-74.

Observatorio Estatal de la Discapacidad (Ed.) (2015). El envejecimiento de las personas con discapacidad en Extremadura. Recuperado de https://observatoriodeladiscapacidad.info/ attachments/article/64/Estudio\%20Envejecimiento\%20PCD\%20Extremadura.pdf

OMS (2002). Active ageing a policy framework. Geneva: World Health Organization.

Park, J., Turnbull, A., y Turnbull, H. (2002). Impacts of poverty on quality of life in families with children with disabilities. Excepcional Children, 68, 151-170.

Rodríguez, A., Verdugo, M.A., y Sánchez, M.C. (2008). Calidad de vida familiar y apoyo para los progenitores de personas con discapacidad intelectual. Revista Siglo Cero, 39(227), 1934.

Schalock, R.L., y Verdugo, M.Á. (2003). Calidad de vida. Manual para profesionales de la salud, educación y servicios sociales. Madrid: Alianza.

Venturiello, M.P. (2014). Los adultos con discapacidad motriz y sus familiares: la organización del hogar, los afectos y el trabajo. Revista Española de Discapacidad, 2(2), 103-120.

Verdugo, M.A., Gómez, L.E., Arias, B., Santamaría, M., Clavero, D., Tamarit, J. (2013): Escala INICO-FEAPS: Evaluación Integral de la Calidad de Vida de personas con Discapacidad Intelectual o del Desarrollo. Salamanca: INICO.

VV.AA. (2004): Necesidades percibidas en el proceso de envejecimiento de las personas con discapacidad intelectual. Revista Siglo Cero, 209.

Recibido: 19 de abril de 2019

Recepción Modificaciones: 27 de abril de 2019

Aceptado: 4 de mayo de 2019 